\title{
Levels of Competence in Procedural Skills
}

\author{
Jon R. Star \\ Combined Program in Education and Psychology; University of Michigan; Ann Arbor, MI 48109-1259 \\ Tel: 734-936-2752, Fax: 734-615-2164 \\ Email: jonstar@umich.edu
}

\begin{abstract}
How do we master procedural skills in domains such as mathematics? Existing models of procedural acquisition fail to distinguish between levels of "skilled" performance. In particular, there is some evidence that automaticity may not be the only endpoint in the acquisition of a skill. Issues of expertise, automaticity, and competency are explored in a case study of a 8th grader in the domain of algebra equation solving. Multiple levels of competence, leading to expertise, are described. I conclude that a learner can become quite accomplished and competent at a procedural skill, and yet fail to display some qualities of expert behavior. In particular, the role of algorithms versus heuristics in the development of competence is highlighted.
\end{abstract}

Keywords: mathematics education, learning theory, cognitive science, middle school

\section{Introduction}

How do we master procedural skills in domains such as mathematics? According to Anderson's model of developing procedural skills, learners progress through a series of three stages -- a declarative stage, a knowledge compilation stage, and a tuning stage (Anderson, 1996).

One criticism of Anderson's model is its inability to distinguish among levels of "skilled" performance. Anderson's theory proposes that the achievement of automaticity is the endpoint in the acquisition of a skill, where a learner's knowledge is fully compiled and s/he is able to fluently execute a skill. But automaticity may not be the only endpoint of procedural skill development. Karmiloff-Smith has found that competent, automatic performance can be followed by a period of meta-procedural representational redescription, where procedural knowledge becomes increasingly explicit, flexible, manipulatable, and available to conscious access (Karmiloff-Smith, 1992).

In this study, I explore the issues of expertise, automaticity, and "skilled" performance through a close analysis of the development of one child's competence in the domain of linear equation solving.

\section{Method}

The subject of this study was a 14-year old, male, 8th grade student, Noah. The experimenter (JS) worked with Noah for 4 sessions over a period of three weeks. Each session lasted approximately 45 minutes. All sessions were videotaped. At the time of the tutoring sessions, Noah had not covered the sections of his symbolic algebra text which dealt with equation solving.

Instruction in equation solving procedures was done by pattern recognition on portions of problems. For example, JS gave Noah the problem step $x+7=12$ and told him a cue to the problem pattern (e.g., "When you see a problem like this,") and then the operator action (e.g., "subtract a 7 from both sides"). Noah was not given instruction which mentioned operator subgoals nor on the order that operators should be applied.

Noah engaged in a two-step verbal protocol during the equation solving portion of the sessions -- planning and solving. In the first step (planning), Noah was asked to verbally describe or plan all problem-solving steps that he thought would be needed to solve a particular problem. Planning occurred before Noah began any written work on the problem. In the second step (solving), Noah attempted to solve the problem. Noah and JS had very little verbal interaction while Noah was engaged in solving.

\section{Results and Discussion}

Three main claims are based on a close examination of the video records of Noah's problem-solving. First, the development of algorithms was an important achievement for Noah, however it was not the culmination of his 
learning. In fact, the abandonment of algorithms in favor of heuristics represents a much more advanced way of approaching equations.

Second, the ability to execute operators individually, which was achieved in the very early stages of Noah's learning, did not enable Noah to use operators in the context of equation solving. Noah had to learn about operators in context, including possible orders of operators and subgoals of operators, before he was able to exhibit knowledge beyond mere operator competence. Similarly, the ability to solve equations of the form $a x+b=c$ using an algorithm did not mean that Noah was necessarily able to solve complex equations such as $5 x+4(x+1)+3=2 x+3(x+4)-1$. Despite the fact that he had achieved fluency on all sub-skills necessary for the solution of such a problem, he was not able to utilize this knowledge to solve more complex problems.

Third, the instruction given to Noah in operator use was purposefully "procedural," in that JS chose not to provide Noah with any "concepts" which underlie the equation solving operators. In the absence of conceptual instruction, Noah was nevertheless able to develop very robust and useful heuristics for solving many different kinds of equations. Noah's understanding of these heuristics involved knowing such things as the order of steps, the subgoals of steps, the types of problems for which certain heuristics may or may not be useful, and the ability to plan to the solution state. Expertise in algebra equation solving would require an even more thorough procedural understanding.

\section{Conclusion}

I conclude that a learner can become quite accomplished and competent at a procedural skill, and yet fail to display some qualities of expert behavior. In addition to fluency in performance, a successful account of procedural learning must include a way to incorporate these more general characteristics of expertise.

\section{References}

Anderson, J. R. (1996). The architecture of cognition. Cambridge, MA: Harvard University Press.

Karmiloff-Smith, A. (1992). Beyond modularity: A developmental perspective on cognitive science. Cambridge, MA: MIT Press. 